\title{
Aplicación del modelo Bristow-Campbell para estimar la irradiación solar global en la zona alto andina de la región Tacna
}

\author{
Application of the Bristow-Campbell model to estimate the global solar irradiation in \\ the high andean area of the Tacna region
}

${ }^{1}$ Lilian Caceres Masco ${ }^{2}$ Hugo torres Muro Jhony Chavez Delgado

\begin{abstract}
RESUMEN
Este trabajo tiene como objetivo, explicar el proceso de determinación de la irradiación solar global para una superficie horizontal en la zona altoandina y andina de la región Tacna a través del modelo empírico propuesto por Bristow - Campbell (1984), y así contar con una base de datos, para ello se recopilo registros diarios de temperatura máxima y mínima de siete estaciones meteorológicas distribuidas en la zona de estudio, durante el periodo 2014-2017. La zona altoandina y andina presenta valores anuales de irradiación solar global superiores a los $5.5 \mathrm{kWh} \mathrm{m}^{-2} \mathrm{~d}^{-1}$, siendo el distrito de Susapaya, provincia de Tarata que presenta el mayor índice de energía solar promedio anual de $6.23 \mathrm{kWh} \mathrm{m}^{-2} \mathrm{~d}^{-1}$ por lo tanto, es viable y rentable las aplicaciones solares en estas zonas, con tecnologías adecuadas para regiones frías.
\end{abstract}

Palabras clave: modelo de Bristow - Campbell, estimación de la irradiación solar global, energía solar, amplitud térmica, constantes empíricas.

\begin{abstract}
|101This work aims to explain the process of determining the global solar irradiation for a horizontal surface in the high Andean and Andean zone of the Tacna region through the empirical model proposed by Bristow - Campbell (1984), and thus have a base of data, for this purpose, daily records of maximum and minimum temperature of seven meteorological stations distributed in the study area were compiled during the 2014-2017 period. The high Andean and Andean zone has annual global solar irradiation values higher than $5.5 \mathrm{kWh} \mathrm{m}^{-2} \mathrm{~d}^{-1}$, being the Susapaya district, Tarata province that has the highest annual average solar energy index of $6.23 \mathrm{kWh}^{-2} \mathrm{~d}^{-1}$, therefore, solar applications in these areas are viable and profitable, with technologies suitable for cold regions.
\end{abstract}

Keywords: Bristow - Campbell model, estimation of global solar irradiation, solar energy, thermal amplitude, empirical constants.

Universidad Nacional Jorge Basadre Grohmann. Tacna -Perú. Email: lilianacaceresmasco@gmail.com

Universidad Nacional Jorge Basadre Grohmann. Tacna-Perú. Email: hugotorres@gmail.com

Universidad Nacional Jorge Basadre Grohmann. Tacna-Perú. Email: jhonychavez@yahoo.es 


\section{INTRODUCCION}

La radiación solar es considerada una de las variables de mayor importancia para los estudios y aplicaciones agroclimáticas debido a que constituye la entrada más significativa de energía en los sistemas físicos y biológicos. Como tal, condiciona una buena parte de los factores climáticos ejerciendo una fuerte influencia en la dinámica de los ecosistemas naturales. Además, hoy en día, la utilización de la radiación solar como energía alternativa se perfila como una solución al problema de la disponibilidad energética que la mayoría de las naciones han debido enfrentar de manera permanente, situación que últimamente ha ido acentuándose debido a la creciente demanda de energía. (Aburto, 2007).

Para el correcto aprovechamiento del recurso solar es fundamental conocer de la forma más precisa posible, la cantidad y la distribución de la radiación solar que incide en un determinado lugar, así como su variación a escala diaria, mensual y anual, para ello se hace uso de los principales instrumentos de medición de la energía solar, sin embargo los lugares en Perú donde se realizan mediciones de esta variable son escasos, debido al costo que representan los equipos y al cuidado que estos necesitan.

Es por ello que desde 1980 se vienen desarrollando modelos numéricos para la estimación de la energía solar, esto como solución a la falta de instrumentos de medición en muchas estaciones meteorológicas. Uno de estos modelos fue propuesto por Bristow Campbell (1984) y fue validado por Baigorria et al. (2003). Entre los modelos probados y utilizados para estimar la radiación solar en función de la temperatura, el modelo Bristow-Campbell se recomendó como el más aplicable a Perú.

La zona de estudio para aplicar este modelo fue la zona altoandina y andina de la región Tacna donde, determinar el recurso solar es de suma importancia, ya que brindan confianza para la aplicación de tecnologías solares a nivel productivo y doméstico.

\section{MODELO DE BRISTOW CAMPBEL}

El modelo de Bristow y Campbell (1984), desarrolla una relación entre transmisividad atmosférica $(\tau)$ o radiación solar relativa $\left(\mathrm{H} / \mathrm{H}_{\mathrm{o}}\right)$ en función de la amplitud térmica $(\Delta T)$.
La explicación física de modelo de Bristow \&Campbell depende de la relación de Bowen, la cual describe que la diferencia entre las temperaturas máximas y mínimas en un día determinado depende de la relación entre el calor sensible y el calor latente. El calor sensible varía de acuerdo a la radiación solar incidente y es responsable de las temperaturas máximas. Durante la noche, el calor sensible es perdido hacia el espacio como radiación en onda larga, disminuyendo así la temperatura del aire hasta su valor mínimo, valor que normalmente se alcanza antes del amanecer (Meza y Varas, 2000).

Utilizando este argumento, Bristow \& Campbell (1984) sugirieron la siguiente relación para la radiación solar global en función de la radiación solar extraterrestre y la diferencia de temperaturas.

$$
\begin{aligned}
& \frac{H}{H_{o}}=a_{B} \cdot\left[1-e^{-b_{B}\left(T_{m a ́ x}-T_{m i ́ n}\right)^{c_{B}}}\right] \\
& \text { Donde: }
\end{aligned}
$$

$H$ : Irradiación solar global [ $\mathrm{Wh} \mathrm{m}^{-2} \mathrm{dia}^{-1} \mathrm{o} \mathrm{MJ} \mathrm{m}^{-2} \mathrm{dia}^{-1}$ ]

$H_{o}$ : Irradiación solar extraterrestre [ $\mathrm{Wh} \mathrm{m}^{-2} \mathrm{dí}^{-1} \mathrm{o} \mathrm{MJ} \mathrm{m}{ }^{-2} \mathrm{dia}^{-1}$ ]

$T_{\text {máx }}$ : Temperatura máxima $\left[{ }^{\circ} \mathrm{C}\right]$

$T_{\text {min }}$ : Temperatura mínima $\left[{ }^{\circ} \mathrm{C}\right]$

$a_{B}, b_{B}$ у $c_{B}:$ Coeficientes empíricos

En otras referencias es muy común encontrar que la diferencia entre la temperatura máxima y mínima, se describe como amplitud térmica ( $\Delta T=T_{\text {máx }}-T_{m i ́ n}$ )

\section{METODOLOGÍA}

La zona de estudio la constituye la zona altoandina y andina de la región Tacna, con una extensión aproximada de $5766.10 \mathrm{~km}^{2}$ la cual abarca gran parte de la provincia de Tarata, Candarave y el distrito de Palca. La base de datos la constituye los registros diarios de temperaturas máximas y mínimas obtenidas de siete estaciones meteorológicas, pertenecientes al Servicio Nacional de Meteorología e Hidrología (Senamhi), en un periodo de registro de 4 años consecutivos, que comprende entre 2014 al 2017, con un total de 20228 datos.

Las coordenadas geográficas de cada una de las estaciones meteorológicas utilizadas, divididas por zonas, siendo la zona altoandina por encima de los 4000 m.s.n.m. y la zona andina entre 2500 a 3900 m.s.n.m., así como la ubicación provincial y distrital. (verTabla 1) 
Aplicación del modelo bristow-campbell para estimar la irradiación solar global en la zona alto andina de la región Tacna

Tabla 1. Estaciones meteorológicas convencionales de Senamhi en la zona altoandina y andina de la región Tacna.

\begin{tabular}{llllccc}
\hline No & Estación & Provincia & Distrito & $\begin{array}{c}\text { Latitud } \\
{\left[{ }^{\circ}\right]}\end{array}$ & $\begin{array}{c}\text { Longitud } \\
{\left[{ }^{\circ}\right]}\end{array}$ & $\begin{array}{c}\text { Altitud } \\
{[\mathrm{m} . \text { s.n.m }]}\end{array}$ \\
\hline & & \multicolumn{2}{c}{ Zona Altoandina } \\
\hline 1 & Chuapalca & Tarata & Tarata & $-17,305$ & $-69,644$ & 4177 \\
2 & Vilacota & Tarata & Susapaya & $-17,129$ & $-70,051$ & 4390 \\
3 & Pampa umalzo & Candarave & Candarave & $-16,875$ & $-70,424$ & 4609 \\
4 & Paucarani & Tacna & Palca & $-17,525$ & $-69,779$ & 4609 \\
\hline & & & Zona Andina & & \\
\hline 5 & Aricota & Candarave & Quilahuani & $-17,326$ & $-70,235$ & 2850 \\
6 & Tarata & Tarata & Tarata & $-17,479$ & $-70,036$ & 3100 \\
7 & Cairani & Candarave & Cairani & $-17,288$ & $-70,339$ & 3920 \\
\hline
\end{tabular}

A través del software ArcGis 10.3, basado en las latitudes y longitudes de las siete estaciones meteorológicas de la red de Senamhi, se obtuvo el mapa de ubicación geográfica de las estaciones utilizadas en la zona alto andina y andina de la región Tacna, la cual se muestra en laFigura 1

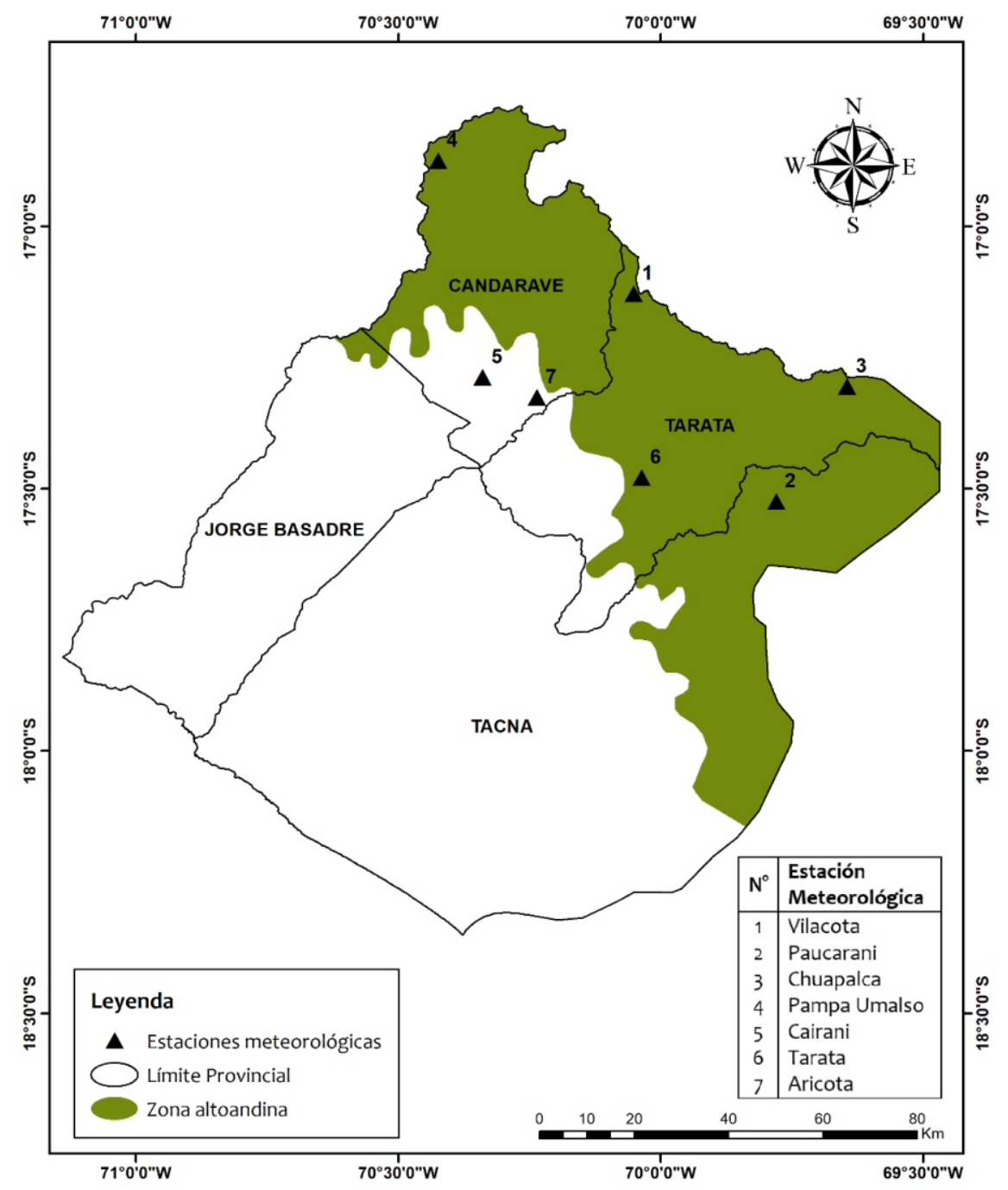




\section{Aplicación del modelo}

A continuación, se muestra la secuencia de ecuaciones necesarias para aplicar el modelo de Bristow-Campbell (1984) para luego ser llevadas a una hoja de cálculo de Excel y así generar una base de datos irradiación solar global para una superficie horizontal.

- Irradiación solar extraterrestre sobre un plano horizontal $\left(H_{o}\right)$

$$
H_{o}=\frac{24}{\pi} I_{s c} E_{o}\left[\cos \delta \cdot \cos \phi \cdot \operatorname{sen} \omega+\operatorname{sen} \delta \cdot \operatorname{sen} \phi \cdot \omega_{s}\right]
$$

- La constante solar $\left(I_{s c}\right)$ es, que equivale a $1362 \mathrm{~W} \mathrm{~m}^{-2}$ o $0.082 \mathrm{MJ} \mathrm{m}^{-2} \mathrm{~min}^{-1}$

- Factor de corrección de la excentricidad de la órbita terrestre $\left(E_{0}\right)$,

$$
\begin{gathered}
E_{0}=\left(\frac{r_{0}}{r}\right)^{2}=1.000110+0.034221 \cos \Gamma+0.001280 \sin \Gamma \\
+0.000719 \cos 2 \Gamma+0.000077 \sin 2 \Gamma \\
\Gamma=\frac{2 \pi\left(d_{n}-1\right)}{365}
\end{gathered}
$$

- Declinación solar $(\boldsymbol{\delta})$

$$
\begin{array}{r}
\delta=(0.006918-0.399912 \cos \Gamma+0.070257 \sin \Gamma-0.006758 \cos 2 \Gamma \\
+0.000907 \sin 2 \Gamma-0.002697 \cos 3 \Gamma+0.00148 \sin 3 \Gamma)
\end{array}
$$

- Ángulo horario $\left(\omega_{s}\right)$

y reemplazando en la ec. (2) obtenemos $\mathrm{H}_{0}$

$$
\omega_{s}=\cos ^{-1} \cdot(-\tan \phi \cdot \tan \delta)
$$

- Constantes empíricas $\left(c_{B}\right)$ y $\left(b_{B}\right)$

$$
\begin{gathered}
c_{B}=2.116-0.0072\left(T_{\text {máx }}-T_{\text {mín }}\right)+57.574 e^{\phi} \\
b_{B}=0.107 c_{B}^{-2.6485}
\end{gathered}
$$

y reemplazando en la ec. (1) obtenemos el resultado para el modelo Bristow \& Campbell

Los datos de entrada para la hoja de Excel son: latitud $(\phi)$, la constante $\boldsymbol{a}_{\boldsymbol{B}}$ y los datos de temperatura máxima y mínima. Para la selección de la constante empírica ab, se utilizó los valores determinado en el atlas solar del Perú (2003), para la selección se consideró al clima y la altitud, siendo para la zona altoandina $a_{B}=0.7$ y para la zona andina $a_{B}=0.76$.

\section{RESULTADOS}

En la Tabla 1. Se presenta el resultado del desarrollo del modelo de Bristow- Campbell en el software Microsoft Excel.

\section{Comportamiento diario de la irradiación solar global}

En laFigura 2, se muestra el comportamiento diario de la irradiación solar global sobre una superficie horizontal, durante el periodo 2014 al 2017 de la estación Paucarani, ubicado en la zona altoandina, provincia de Tacna, distrito de Palca y a una altitud de 4609 msnm y de la estación Aricota ubicado en la zona andina, provincia de Candarave distrito de Quilahuani a una altura de $2850 \mathrm{msnm}$.

\section{Comportamiento mensual de la irradiación solar global}

En laFigura 3. se muestra el comportamiento mensual de la irradiación solar global, en la zona alto altoandina y andina de la región Tacna, para el periodo 2014 al 2017. Se observa que la irradiación mínima en las siete estaciones es de $4.21 \mathrm{kWh} \mathrm{m}^{-2} \mathrm{~d}^{-1}$ correspondiente a la estación de Paucarani en el distrito de Palca y un máximo de $7.66 \mathrm{kWh} \mathrm{m}^{-2} \mathrm{~d}^{-1}$ en la estación Vilacota de la provincia de Tarata.

\section{Promedios anuales de la irradiación solar global}

En la Tabla 2, se describe el comportamiento de la irradiación solar global promedio anual, para cada estación meteorológica de estudio durante el periodo 2014 al 2017. 
Aplicación del modelo bristow-campbell para estimar la irradiación solar global en la zona alto andina de la región Tacna

Tabla 2. Desarrollo del Modelo de Bristow-Campbell en Excel para estimar la irradiación solar global diaria en la estación Paucarani correspondiente al mes de enero del 2015.

\begin{tabular}{|c|c|c|c|c|c|c|c|c|c|c|c|}
\hline \multicolumn{2}{|c|}{ ESTACIEN: } & \multirow[b]{2}{*}{ 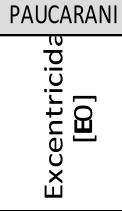 } & \multirow[b]{2}{*}{ 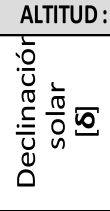 } & \multirow[b]{2}{*}{ 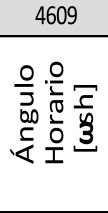 } & \multirow[b]{2}{*}{ 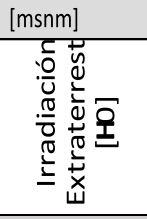 } & \multirow[b]{2}{*}{ 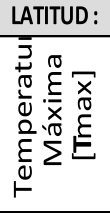 } & \multicolumn{2}{|c|}{$-0,3059$ [rad] } & \multicolumn{2}{|c|}{ CONSTANTE B-C [aB] : } & \multirow[b]{2}{*}{ 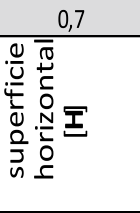 } \\
\hline 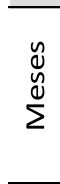 & $\begin{array}{l}0 \\
\stackrel{2}{\pi} \\
\frac{\pi}{0} \\
\stackrel{0}{0}\end{array}$ & & & & & & 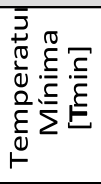 & 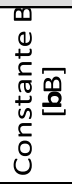 & $\begin{array}{l}\infty \\
0 \\
0 \\
\frac{1}{0} \\
0 \\
0 \\
0 \\
0 \\
0 \\
0\end{array}$ & 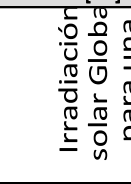 & \\
\hline$[-]$ & {$[-]$} & {$[-]$} & [rad] & [rad] & {$\left[\mathrm{MJ} \mathrm{m} \mathrm{m}^{-2} \mathrm{dia}^{-1}\right]$} & {$\left[{ }^{\circ} \mathrm{C}\right]$} & {$\left[{ }^{\circ} \mathrm{C}\right]$} & {$[-]$} & {$[-]$} & {$\left[\mathrm{MJ} \mathrm{m}^{-2} \mathrm{di}^{-1}{ }^{-1}\right]$} & Wh m $\left.\mathrm{m}^{-2} \mathrm{di}^{-1}\right]$ \\
\hline \multirow{31}{*}{$\begin{array}{l}\frac{O}{d} \\
\frac{1}{ \pm} \\
\end{array}$} & 1 & 1,0351 & $-0,4024$ & 1,7056 & 41,6601 & 10,9 & 1,0 & 0,0436 & 1,4032 & 19,3422 & 5,3728 \\
\hline & 2 & 1,0351 & $-0,4011$ & 1,7051 & 41,6553 & 13,6 & $-2,4$ & 0,1179 & 0,9640 & 23,8710 & 6,6308 \\
\hline & 3 & 1,0351 & $-0,3995$ & 1,7045 & 41,6495 & 16,3 & $-4,2$ & 0,3489 & 0,6400 & 26,5393 & 7,3720 \\
\hline & 4 & 1,0351 & $-0,3979$ & 1,7039 & 41,6426 & 13,9 & 2,5 & 0,0539 & 1,2952 & 20,8908 & 5,8030 \\
\hline & 5 & 1,0351 & $-0,3961$ & 1,7032 & 41,6346 & 10,4 & 0,9 & 0,0413 & 1,4320 & 18,8291 & 5,2303 \\
\hline & 6 & 1,0350 & $-0,3942$ & 1,7025 & 41,6254 & 12,2 & 2,6 & 0,0419 & 1,4248 & 18,9542 & 5,2650 \\
\hline & 7 & 1,0350 & $-0,3922$ & 1,7018 & 41,6152 & 12,5 & $-0,2$ & 0,0658 & 1,2016 & 21,9087 & 6,0857 \\
\hline & 8 & 1,0350 & $-0,3900$ & 1,7010 & 41,6037 & 11,4 & 1,4 & 0,0442 & 1,3960 & 19,4350 & 5,3986 \\
\hline & 9 & 1,0349 & $-0,3877$ & 1,7001 & 41,5911 & 13,0 & 0,8 & 0,0608 & 1,2376 & 21,5271 & 5,9798 \\
\hline & 10 & 1,0348 & $-0,3853$ & 1,6992 & 41,5772 & 14,9 & $-1,0$ & 0,1156 & 0,9712 & 23,7736 & 6,6038 \\
\hline & 11 & 1,0347 & $-0,3827$ & 1,6983 & 41,5621 & 15,9 & $-1,7$ & 0,1652 & 0,8488 & 24,6728 & 6,8536 \\
\hline & 12 & 1,0347 & $-0,3800$ & 1,6973 & 41,5457 & 14,0 & $-1,8$ & 0,1134 & 0,9784 & 23,7029 & 6,5841 \\
\hline & 13 & 1,0346 & $-0,3772$ & 1,6962 & 41,5281 & 14,6 & $-2,4$ & 0,1448 & 0,8920 & 24,3266 & 6,7574 \\
\hline & 14 & 1,0344 & $-0,3743$ & 1,6952 & 41,5090 & 12,8 & $-1,9$ & 0,0923 & 1,0576 & 23,0903 & 6,4140 \\
\hline & 15 & 1,0343 & $-0,3713$ & 1,6941 & 41,4887 & 14,5 & $-2,6$ & 0,1480 & 0,8848 & 24,3571 & 6,7659 \\
\hline & 16 & 1,0342 & $-0,3681$ & 1,6929 & 41,4669 & 8,9 & 0,1 & 0,0377 & 1,4824 & 17,7754 & 4,9376 \\
\hline & 17 & 1,0340 & $-0,3648$ & 1,6917 & 41,4437 & 11,4 & $-0,3$ & 0,0564 & 1,2736 & 21,0498 & 5,8472 \\
\hline & 18 & 1,0339 & $-0,3614$ & 1,6905 & 41,4190 & 9,8 & $-0,4$ & 0,0455 & 1,3816 & 19,5784 & 5,4384 \\
\hline & 19 & 1,0337 & $-0,3579$ & 1,6892 & 41,3929 & 14,0 & $-1,3$ & 0,1030 & 1,0144 & 23,3512 & 6,4864 \\
\hline & 20 & 1,0335 & $-0,3543$ & 1,6879 & 41,3652 & 12,4 & $-0,4$ & 0,0668 & 1,1944 & 21,8469 & 6,0686 \\
\hline & 21 & 1,0334 & $-0,3506$ & 1,6865 & 41,3360 & 15,6 & $-0,7$ & 0,1252 & 0,9424 & 23,8450 & 6,6236 \\
\hline & 22 & 1,0332 & $-0,3467$ & 1,6851 & 41,3051 & 11,5 & $-0,9$ & 0,0628 & 1,2232 & 21,5293 & 5,9804 \\
\hline & 23 & 1,0330 & $-0,3428$ & 1,6837 & 41,2727 & 8,8 & $-0,2$ & 0,0387 & 1,4680 & 17,9845 & 4,9957 \\
\hline & 24 & 1,0327 & $-0,3387$ & 1,6823 & 41,2386 & 11,4 & $-0,6$ & 0,0590 & 1,2520 & 21,1894 & 5,8859 \\
\hline & 25 & 1,0325 & $-0,3345$ & 1,6808 & 41,2028 & 10,4 & $-0,8$ & 0,0524 & 1,3096 & 20,4902 & 5,6917 \\
\hline & 26 & 1,0323 & $-0,3303$ & 1,6793 & 41,1654 & 12,8 & $-1,7$ & 0,0890 & 1,0720 & 22,7882 & 6,3301 \\
\hline & 27 & 1,0320 & $-0,3259$ & 1,6777 & 41,1261 & 13,5 & $-4,8$ & 0,1942 & 0,7984 & 24,8068 & 6,8908 \\
\hline & 28 & 1,0318 & $-0,3214$ & 1,6761 & 41,0851 & 13,9 & $-6,2$ & 0,3105 & 0,6688 & 25,9057 & 7,1960 \\
\hline & 29 & 1,0315 & $-0,3168$ & 1,6745 & 41,0423 & 15,2 & $-4,7$ & 0,2935 & 0,6832 & 25,7449 & 7,1514 \\
\hline & 30 & 1,0312 & $-0,3122$ & 1,6729 & 40,9976 & 14,0 & $-3,5$ & 0,1615 & 0,8560 & 24,2834 & 6,7454 \\
\hline & 31 & 1,0309 & $-0,3074$ & 1,6712 & 40,9511 & 14,0 & $-3,4$ & 0,1580 & 0,8632 & 24,2018 & 6,7227 \\
\hline
\end{tabular}
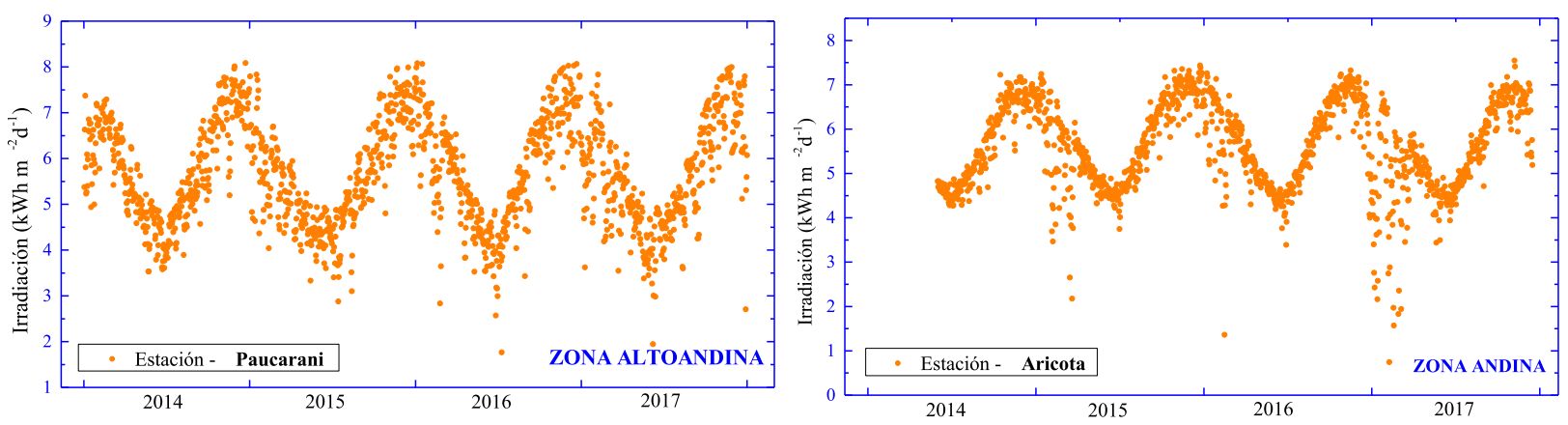

(a)

(b) 
Figura 2. Irradiación solar global diaria durante 2014 al 2017,(a) Estación Paucarani, (b) Estación Aricota

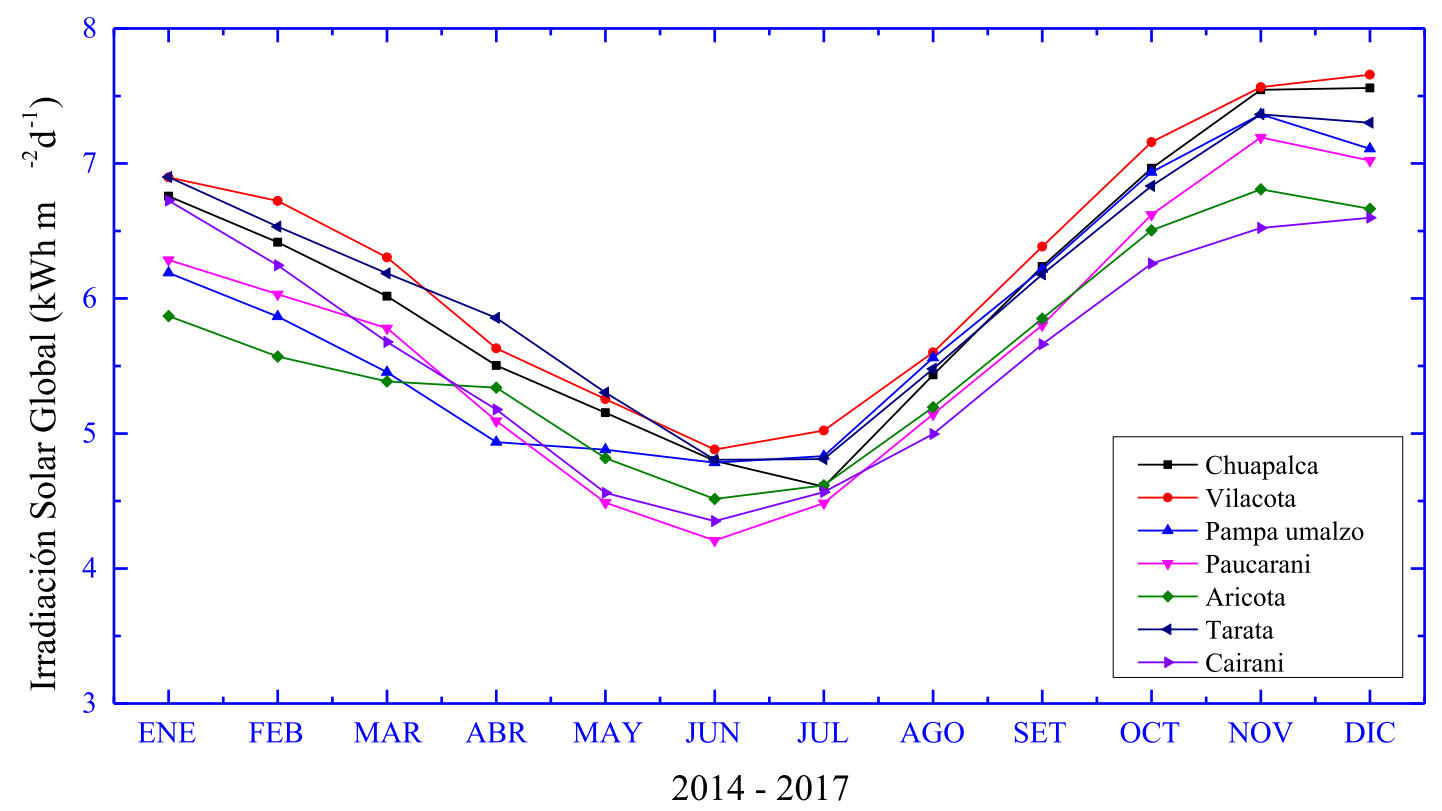

Tabla 2.

Irradiación solar global promedio anual en la zona altoandina y andina de la región Tacna.

\begin{tabular}{|c|c|c|c|c|c|}
\hline \multirow{2}{*}{$\mathbf{N}^{\circ}$} & \multirow{2}{*}{ Estación } & \multicolumn{4}{|c|}{ Irradiación Solar Global $\left[\mathrm{kWh} \mathrm{m}^{-2} \mathrm{~d}^{-1}\right]$} \\
\hline & & 2014 & 2015 & 2016 & 2017 \\
\hline & & \multicolumn{4}{|c|}{ Zona Altoandina } \\
\hline 1 & Chuapalca & 6,03 & 6,10 & 6,31 & 6,06 \\
\hline 2 & Vilacota & 6,28 & 6,11 & 6,33 & 6,20 \\
\hline 3 & Pampa umalzo & 5,89 & 5,79 & 5,54 & 5,72 \\
\hline \multirow[t]{2}{*}{4} & Paucarani & 5,81 & 5,54 & 5,76 & 5,59 \\
\hline & & \multicolumn{4}{|c|}{ Zona Andina } \\
\hline 5 & Aricota & 5,67 & 5,66 & 5,73 & 5,35 \\
\hline 6 & Tarata & 6,13 & 6,07 & 6,30 & 6,03 \\
\hline 7 & Cairani & 5,87 & 5,81 & 5,66 & 5,10 \\
\hline
\end{tabular}

\section{Mapa solar anual de la zona altoandina y andina de la región Tacna}

En la Tabla 3, se muestra los promedios de la irradiación solar global para un plano horizontal correspondiente a los 4 años de estudio, que comprende desde el 2014 al 2017, para cada estación de registro, así como también su ubicación provincial y distrital.

Siendo el máximo valor de irradiación solar global anual de $6,23 \mathrm{kWh} \mathrm{m}^{-2} \mathrm{~d}^{-1}$ de la estación meteorológica de Vilacota pertenecientes a la provincia de Tarata, distrito de Susapaya y la mínima de 5,60 $\mathrm{kWh} \mathrm{m}^{-2} \mathrm{~d}^{-1}$ que corresponde a la estación de Aricota, provincia de Candarave.

Por otro lado, las estaciones ubicadas en la provincia de Tarata presentan valores superiores a los $6 \mathrm{kWh} \mathrm{m}^{-2} \mathrm{~d}^{-1} \mathrm{y}$ las estaciones ubicadas en la provincia de Candarave tienden a valores no mayores de $5.74 \mathrm{kWh} \mathrm{m}^{-2} \mathrm{~d}^{-1}$ anual, lo que representa que existe una similitud de energía solar por zona provincial, no dependiendo de la altitud que se encuentren. 
Aplicación del modelo bristow-campbell para estimar la irradiación solar global en la zona alto andina de la región Tacna

Tabla 3. Promedio anual de la irradiación solar global sobre una superficie horizontal para cada estación meteorológica del periodo 2014-2017.

\begin{tabular}{|c|c|c|c|c|}
\hline \multirow{2}{*}{$\mathbf{N}^{\circ}$} & \multirow{2}{*}{ Estación } & \multirow{2}{*}{ Provincia } & \multirow{2}{*}{ Distrito } & \multirow{2}{*}{$\begin{array}{c}\text { Irradiación Solar Global } \\
\left\lceil\mathrm{kWh} \mathrm{m}^{-2} \mathrm{~d}^{-1}\right\rceil \\
\end{array}$} \\
\hline & & & & \\
\hline \multicolumn{5}{|c|}{ Zona Altoandina } \\
\hline 1 & Chuapalca & Tarata & Tarata & 6,12 \\
\hline 2 & Vilacota & Tarata & Susapaya & 6,23 \\
\hline 3 & Pampa umalzo & Candarave & Candarave & 5,74 \\
\hline 4 & Paucarani & Tacna & Palca & 5,68 \\
\hline \multicolumn{5}{|c|}{ Zona Andina } \\
\hline 5 & Aricota & Candarave & Quilahuani & 5,60 \\
\hline 6 & Tarata & Tarata & Tarata & 6,13 \\
\hline 7 & Cairani & Candarave & Cairani & 5,61 \\
\hline
\end{tabular}

Los promedios de irradiación solar global superan los $5,5 \mathrm{kWh} \mathrm{m}^{-2} \mathrm{~d}^{-1}$ anual, tanto para la zona altoandina y andina de la región Tacna; lo que representa alta rentabilidad para las aplicaciones solares según la
OLADE (Organización Latinoamericana de Energía). Con los promedios anuales de los cuatros de estudio, se realizó un mapa solar de la zona altoandina de la región Tacna (ver figura 4)

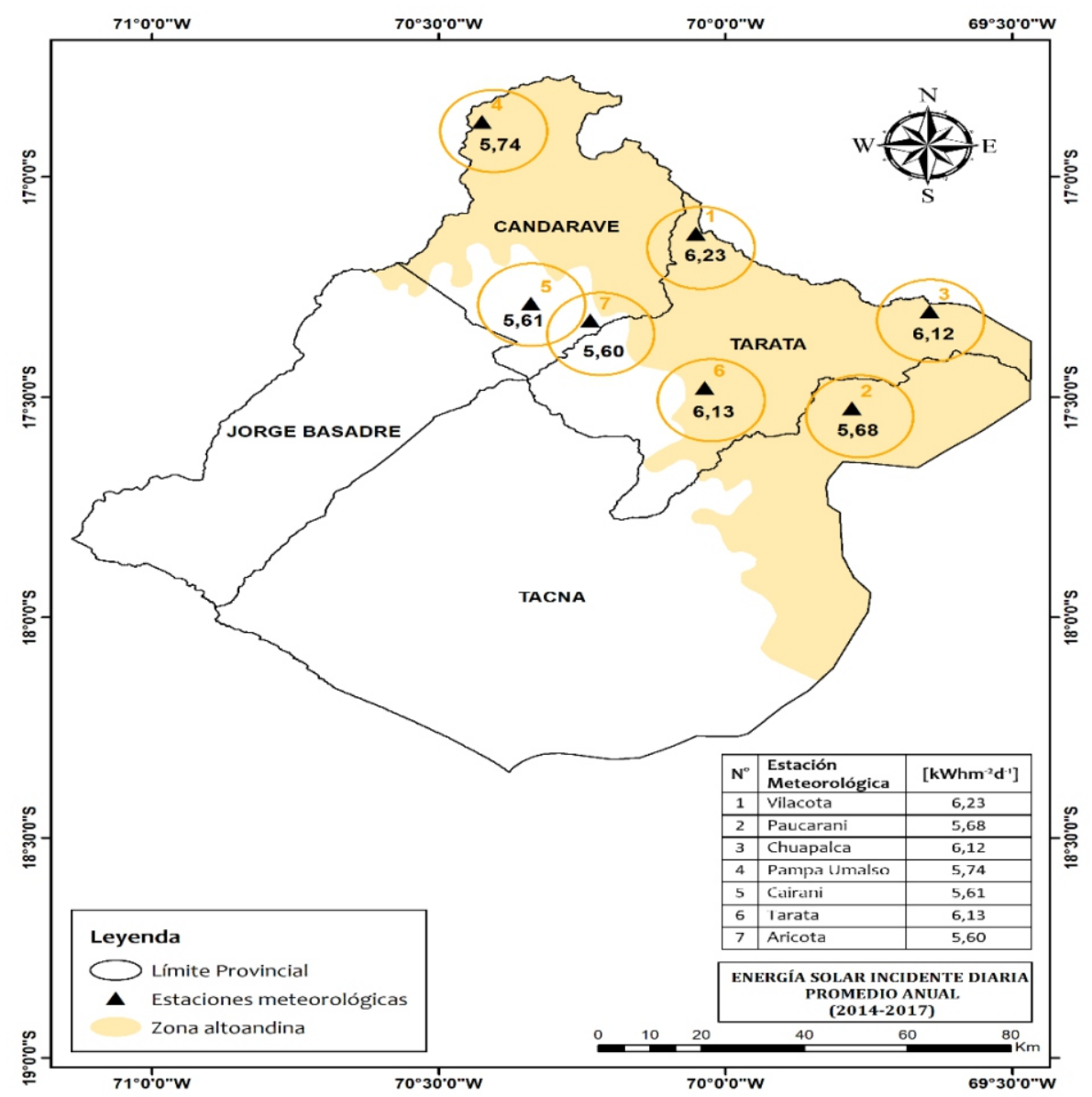

Figura 4. Mapa solar de la zona altoandina y andina de la región Tacna 


\section{Irradiancia solar global y amplitud térmica}

En la figura 5, en (a), (b) y (c) se muestra la relación entre la irradiación solar global y la amplitud térmica, recordando que la amplitud térmica se describe como la diferencia entre la temperatura máxima y mínima ( $\Delta T=T_{\max }-$ $\left.T_{\min }\right)$, para tres estaciones en diferentes zonas.

En la gráfica se observa que la amplitud térmica para la estación Paucarani correspondiente a la zona altoandina de la región Tacna, llega a un máximo de $29^{\circ} \mathrm{C}$, siendo estos durante los meses de invierno, debido al tipo de clima en la zona alta, a diferencia de la estación Aricota ubicado en la zona andina, la amplitud térmica no supera los $20^{\circ} \mathrm{C}$, pero ambas graficas tienen un comportamiento similar a la curva gaussiana, a diferencia de la estación del CERT (Centro de Energías Renovables Tacna) ubicado en la zona costa de la región, la amplitud térmica máxima no supera los $15^{\circ} \mathrm{C}$, y su comportamiento es inverso a la curva gaussiana.

La relación entre la irradiación solar global y la amplitud térmica según las gráficas es inversamente proporcional, esto para la zona altoandina y andina, ya que para la estación del CERT en zona costa la relación es directamente proporcional.

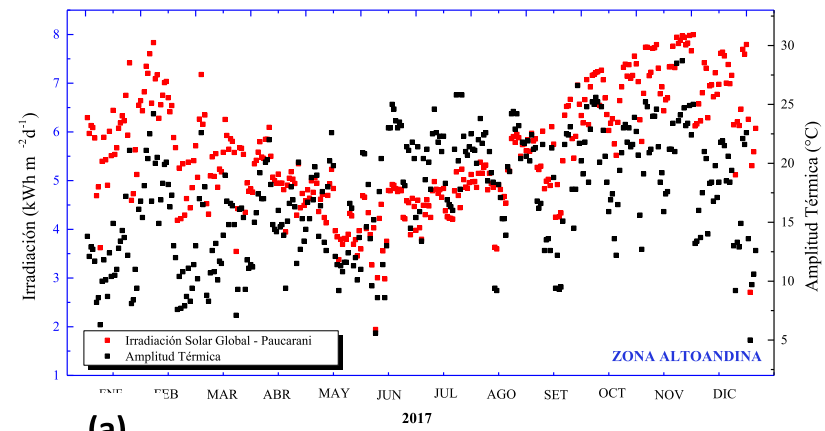

(a)

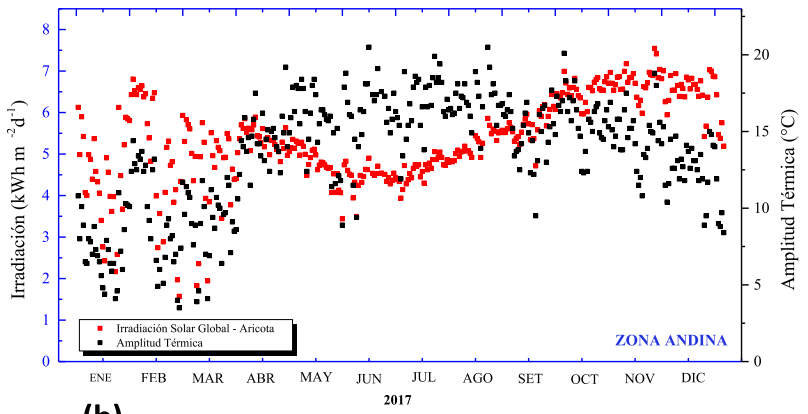

(b)

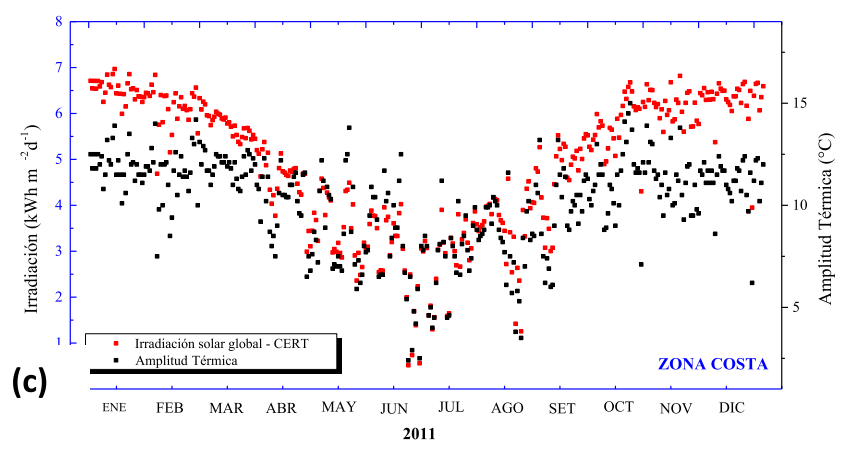

En la figura 6. se muestra la gráfica de las ecuaciones de las constantes empíricas $\left(b_{B}\right)$ y $\left(c_{B}\right)$, se observa que a medida que la amplitud térmica crece, la constante $\left(c_{B}\right)$ se hace más pequeño; llegando a ser negativo, todo lo contrario, sucede con la constante $\left(b_{B}\right)$ que a medida que crece la amplitud térmica esta toma valores mayores.

\section{Comparación entre datos estimados y medidos de la irradiación solar global}

Debido a que en la zona de estudio no cuenta con equipos de medición de energía solar, se consideró los registros del CERT (Centro de energías renovables de Tacna) ubicado en la zona costa de la región, para realizar una comparación entre datos, sin embargo, esta estación no se encuentra en la zona de estudio, pero la finalidad es comparar datos.

En la figura 7, se muestra el comportamiento entre los datos estimados de la irradiación solar global diaria, mediante registros de temperatura máxima y mínima de Senamhi y los registros de medición del Centro de Energías Renovables de Tacna (CERT), durante el año 2011 en la ciudad de Tacna. Como se observa los datos estimados y medidos son similares, lo que indica que el modelo aplicado es confiable para esta región. 


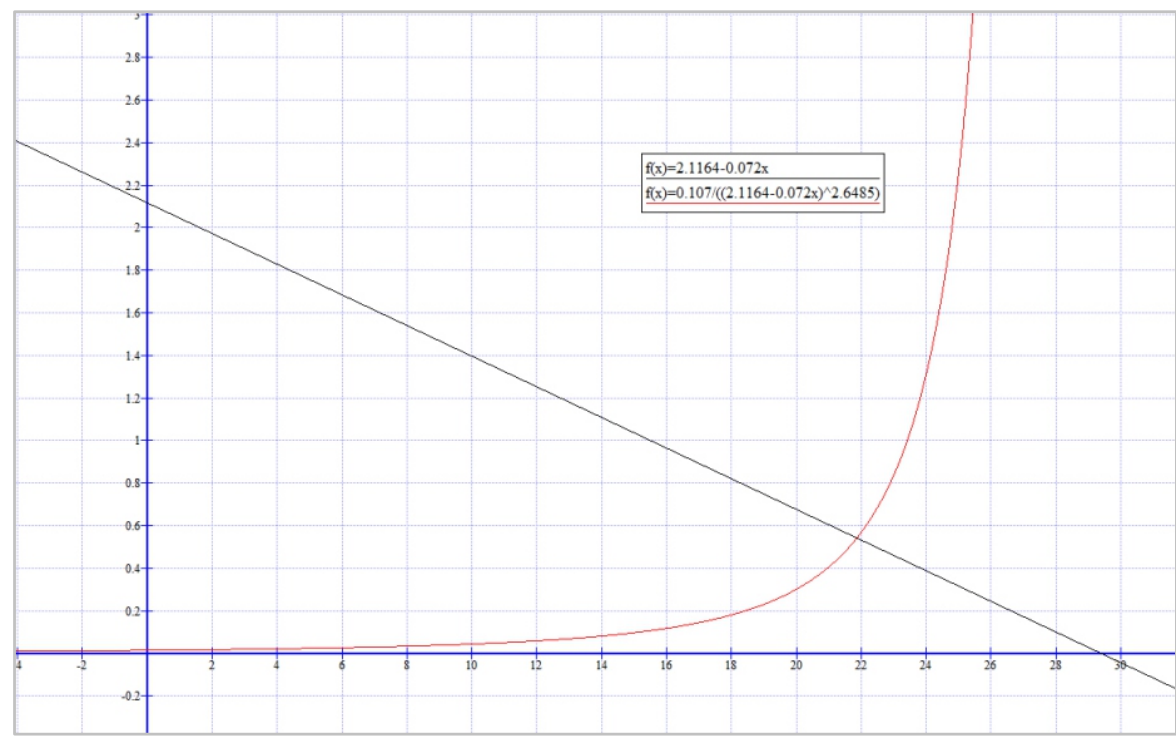

Tabla 3. Promedio anual de la irradiación solar global sobre una superficie horizontal para cada estación meteorológica del periodo 2014-2017.

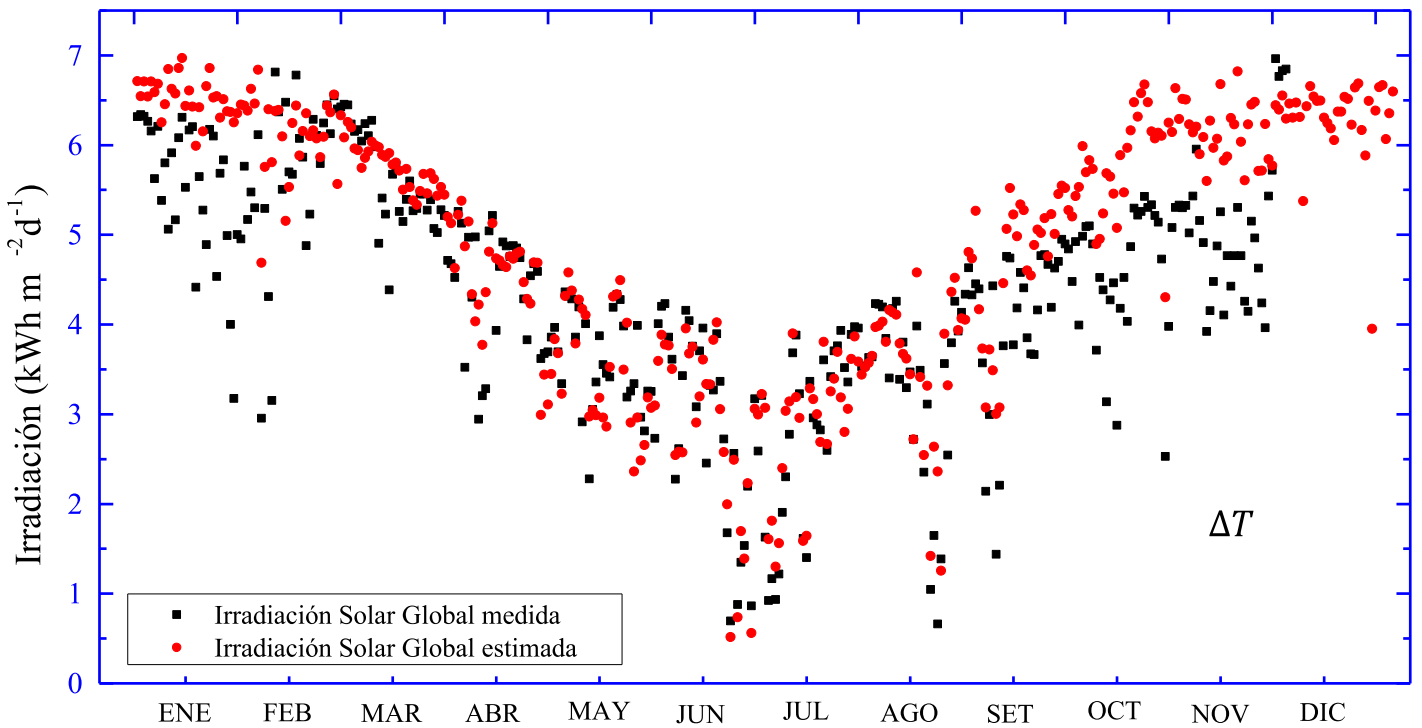

Figura 7. Irradiación solar global diaria estimada y medida en el CERT, correspondiente al año 2011.

\section{DISCUSIÓN}

En la figura 5. Se observa que el comportamiento de la amplitud térmica no es igual para cualquier zona geográfica, mientras que en la zona altoandina llega a valores máximos de hasta $34^{\circ} \mathrm{C}$, en la zona andina llega hasta $\operatorname{los} 20^{\circ} \mathrm{C}$ y en la estación del CERT parte costa de la región no supera $\operatorname{los} 15^{\circ} \mathrm{C}$ y durante los meses de invierno la amplitud térmica en la zona altoandina y andina llega a sus valores máximos mientras que en la zona costa llega a valores mínimos, esto se debe al tipo de clima.
Al graficar las ecuaciones de las constantes empírica $\left(b_{B}\right)$ y $\left(c_{B}\right)$. se observa que la ecuación de $\left(c_{B}\right)$, tiene un límite máximo de amplitud térmica de $29,4^{\circ} \mathrm{C}$, si la amplitud térmica supera este límite, la constante $\left(c_{B}\right)$ se hace negativo, haciendo incalculable la ecuación $\left(b_{B}\right)$, esto implica que estas ecuaciones trabajan con un rango de amplitud térmica, siendo el límite los $29,4^{\circ} \mathrm{C}$. La zona altoandina durante los meses de invierno presenta valores de amplitud térmica de hasta $34^{\circ} \mathrm{C}$ diarios, superando este límite en consecuencia 
durante esos días no se podrá estimar datos diarios de irradiación solar global, y solo datos medios mensuales todo lo contrario, es para la zona andina de Tacna, donde la amplitud térmica no supera $\operatorname{los} 20^{\circ} \mathrm{C}$, estando dentro del rango permitido por las ecuaciones, permitiendo la estimación diaria de la irradiación solar global.

Como una solución para esta limitación en el cálculo, es calibrar las ecuaciones de las constantes empíricas $\left(b_{B}\right)$ y $\left(c_{B}\right)$, de tal manera que permita ampliar el rango de la amplitud térmica, sobre todo para la aplicación en la zona altoandina donde la amplitud térmica diaria llega a valores máximos de hasta $34^{\circ} \mathrm{C}$ esto en días atípicos en los meses de invierno, un ejemplo parecido es el estudio realizado por Gavilán, (s.f) donde también encontró esta limitación en el cálculo por el rango de la amplitud térmica, pero en su caso fue contrario al nuestro, su zona de estudio presentaba valores menores a $12^{\circ} \mathrm{C}$ de amplitud térmica, como solución calibraron las ecuaciones de las constantes con datos medidos y de esa manera ampliaron el rango de la amplitud térmica y se pudo estimar valores de irradiación solar global en esos días donde la amplitud térmica era mínima. Recordar que estas ecuaciones están limitadas a la zona geográfica de aplicación, es decir las ecuaciones de las constates empíricas que se hicieron en el atlas solar del Perú, no son las mismas que las de otros países, al calibrar las ecuaciones que se tienen por regiones (costa, sierra y selva), ya que el comportamiento de la amplitud térmica no es igual para estas tres regiones geográficas, se podrá tener una mejor precisión en la estimación de la irradiación solar global.

Los valores estimados de irradiación solar global para una superficie horizontal para la zona altoandina y andina se encuentran dentro del rango determinado en el atlas solar del Perú (2003) para la región sierra de 5,5-6,5 $\mathrm{kWh} \mathrm{m}^{-}$ ${ }^{2} d^{-1}$, confirmando que la zona sierra tanto baja y alta de Tacna tienen alto potencial solar, siendo el distrito de Susapaya, provincia de Tarata, presenta el mayor índice de energía solar promedio anual de 6,23 $\mathrm{kWh} \mathrm{m}^{-2} \mathrm{~d}^{-1}$ y el distrito de Aricota, provincia de Candarave con el mínimo de 5,61 $\mathrm{kWh} \mathrm{m}^{-2} \mathrm{~d}^{-1}$.

\section{CONCLUSIONES}

El modelo empírico Bristow- Campbell permite determinar la irradiación solar global diaria para un plano horizontal en la zona andina y la media diaria mensual para la zona altoandina de la región Tacna.

La zona altoandina y andina presenta valores anuales de irradiación solar global superiores a los 5,5 $\mathrm{kWh} \mathrm{m}^{-2} \mathrm{~d}^{-1}$, siendo el distrito de Susapaya, provincia de Tarata que presenta el mayor índice de energía solar promedio anual de $6,23 \mathrm{kWh} \mathrm{m}^{-2} \mathrm{~d}^{-1}$ por lo tanto, es viable y rentable las aplicaciones solares en estas zonas, con tecnologías adecuadas para regiones frías.

Los mayores índices de irradiación solar global se encuentran en la provincia de Tarata y los menores en la provincia de Candarave.

Las ecuaciones de las constantes empíricas tienen un límite de amplitud térmica siendo $29,4^{\circ} \mathrm{C}$, cuando la amplitud térmica supera este límite, se presentará limitaciones en el cálculo.

\section{RECOMENDACIONES}

Se recomienda ampliar la red de estaciones meteorológicas e instrumentación piranométrico, el cual nos permitirá la mejora y la validación de los modelos empíricos de recursos solares.

Se recomienda la calibración de las ecuaciones empíricas $\left(b_{B}\right)$ y $\left(c_{B}\right)$, para cada región geográfica (costa, sierra y selva), ya que el comportamiento de la amplitud térmica es distinto para estrás tres regiones y así estimar datos más precisos de energía solar.

\section{REFERENCIAS}

BAIGORRIA, G. A., Villegas, E. B., Trebejo, I., Carlos, J. F., \& Quiroz, R. (2004). Atmospheric transmissivity: distribution and empirical estimation around the central Andes. International Journal of Climatology, 24(9), 1121-1136.

BRISTOW, K. L., \& Campbell, G. S. (1984). On the relationship between incoming solar radiation and daily maximum and minimum temperature. Agricultural and forest meteorology, 31(2), 159-166. 
Aplicación del modelo bristow-campbell para estimar la irradiación solar global en la zona alto andina de la región Tacna

CAMAYO-LAPA, B. F., Pomachagua-Paucar, J. E., Massipe-Hernaández, J. R., Quispe-Flores, M. O., \& Torres-Ten, A. (2017). Validación y aplicación del modelo Bristow Campbell para estimar la radiación solar global de la región de Junín. Tecnología Química, 37(3), 574-590.

IQBAl, M. (1983). An introduction to solar radiation. Toronto, New York: Academic Press.

MEZA, F., \& Varas, E. (2000). Estimation of mean monthly solar global radiation as a function of temperature. Agricultural and Forest Meteorology, 100(2-3), 231-241.

SENAMHI, (2003). Atlas de energía solar del Perú. Lima-Perú: Proyecto PER98/G31-MINEM
ABURTO, C. (2007). Elaboración de un modelo de estimación de la distribución espacial de la radiación solar global mensual para Chile central. (tesis de pregrado). Universidad de Chile, Chile.

LORENZO, E., \& Araujo, G. L. (1994). Electricidad solar: Ingeniería de los sistemas fotovoltaicos (1aㅡ ed.). Madrid: Instituto de Energía Solar.

GAVILÁN, P. (s.f) Modelos De Estimación De La Radiación Solar Diaria A Partir De Medidas De La Temperatura. 